\title{
Circular RNAs: A novel target among non-coding RNAs with potential roles in malignant tumors (Review)
}

\author{
WEISONG ZHAO $^{1^{*}}$, MAN DONG $^{2 *}$, JINRU PAN $^{1}$, YAJIE WANG $^{1}$, \\ JINGYI ZHOU ${ }^{1}$, JIANJUN MA ${ }^{1}$ and SHAOYANG LIU ${ }^{3}$ \\ ${ }^{1}$ Human Anatomy Laboratory, School of Basic Medicine, Xinxiang Medical University; \\ ${ }^{2}$ Department of Medicine, The Third Affiliated Hospital of Xinxiang Medical University, Xinxiang, Henan 453003; \\ ${ }^{3}$ Department of Orthopedics, Shanghai Putuo District Central Hospital, Shanghai 200062, P.R. China
}

Received January 2, 2019; Accepted June 24, 2019

DOI: $10.3892 / \mathrm{mmr} .2019 .10637$

\begin{abstract}
Circular RNAs (circRNAs) are a class of non-coding RNAs that are generated via alternative back-splicing, which connects the terminal 5' and 3'ends. Due to their unique loop structure, circRNAs are resistant to ribonucleases and more stable than linear RNAs. In vivo, they are usually highly conserved and stably expressed in tissue/developmental-stage-specific manners. Generally, circRNAs function as microRNA sponges and splicing regulators, as well as in protein binding and transcription. Some circRNAs contain open reading frames with internal ribosomal entry site elements and can thus encode specific proteins. Previously, circRNAs were thought to be erroneous splicing products or by-products of mRNA splicing. With the development of the next-generation sequencing techniques, it has become increasingly clear that circRNAs are abundantly widespread in eukaryotes and that they play significant roles in malignant tumor progression. The present review briefly introduces the biogenesis and functions of circRNAs, as well as summarizes recent research in several common malignancies. The present review also addresses the prospects of circRNAs in clinical applications.
\end{abstract}

Correspondence to: Mr. Jianjun Ma, Human Anatomy Laboratory, School of Basic Medicine, Xinxiang Medical University, 601 Jinsui Avenue, Xinxiang, Henan 453003, P.R. China

E-mail: mjj19631030@163.com

Mr. Shaoyang Liu, Department of Orthopedics, Shanghai Putuo District Central Hospital, 146 Lanxi Road, Shanghai 200062 , P.R. China

E-mail: shaoyang7817@126.com

${ }^{*}$ Contributed equally

Key words: circular RNAs, microRNA, malignant tumor, biomarker, therapy

\section{Contents}

1. Background

2. Biogenesis and classification of circRNAs

3. Biological functions of circRNAs

4. circRNAs in malignant tumors

5. Clinical applications of circRNAs

6. Conclusions and perspective

\section{Background}

Non-coding RNAs (ncRNAs) are a large group of RNA families that are derived from different genomes but are not translated into proteins (1). Based on their length, they are divided into two categories: Long ncRNAs, which contain $>200$ nucleotides, and short ncRNAs, which contain $<200$ nucleotides; the latter group consists mainly of piwi-interacting RNAs, microRNAs (miRNAs/miRs) and small nucleolar RNAs (2). Accumulating evidence indicated that ncRNAs play crucial roles in a wide variety of vital biological processes, including tumorigenesis, neurological functions, cardiovascular functions and development (3). As a new species of ncRNAs, circular RNAs (circRNAs) are stable, abundant and highly conserved RNA molecules in mammalian cells (4). As previously reported, linear mRNAs are usually more highly expressed in most tissues than circRNAs, while circRNAs are more abundant in brain tissue than linear mRNAs (5). Compared with the expression levels of corresponding linear mRNAs, the expression levels of circRNAs may be up to ten times higher (4). Although circRNAs were first identified in early 1976 (6), they were initially thought to be errors of the splicing process or by-products of mRNA splicing (7). In 2012, Salzman et al (8) first reported that numerous human genes could express circRNAs based on the results of high-throughput sequencing (RNA-seq) analysis (4,7). Later, Jeck et al (4) detected $>25,000$ distinct circRNAs in human fibroblasts by performing RNA-seq experiments with libraries prepared from ribosome-depleted RNA (4). In the same year, Memczak et al (7) developed and published a new computational pipeline that can find circRNAs in any genomic region, and they subsequently identified $\sim 2,000$ human, 700 nematode and 1,900 mouse circRNAs. 
Since then, circRNAs have attracted increasing attention, providing novel insight on circRNAs.

circRNAs are mainly generated by alternative splicing of pre-mRNAs (7). Typically, an upstream splice acceptor is joined to a downstream splice donor, thus forming a covalently closed continuous loop structure $(7,9,10)$. Notably, circRNAs can differ greatly in length. Some consist of exonic or intronic sequences and are $<200 \mathrm{nt}$ or even $<100 \mathrm{nt}$. However, most reported circRNAs are $>200 \mathrm{nt}$ (11). In the last decade, many previous studies have found several characteristics of circRNAs (7-10): i) Abundance and diversity, by taking advantage of RNA-seq technology, thousands of different circRNAs have been identified in eukaryotes, and the complicated mechanism by which circRNAs are generated leads to diversity in the circRNAs formed; ii) stability, due to their unique loop structure, circRNAs are resistant to ribonucleases, rendering them more stable than linear RNAs; iii) conservation, the expression patterns of circRNA are highly conserved in different species, such as plants, mice, zebrafish and humans; and iv) specificity, circRNAs are usually specifically expressed in tissue/developmental-stage-specific manners.

The identification and validation of the presence of particular circRNAs requires specific biochemical approaches. At present, reverse transcription-quantitative PCR (RT-qPCR), one of the most basic detection tools, has been used to detect the relative abundances of circRNAs in biological samples (12). Furthermore, some algorithms, including KNIFE,PTESFinder, CIRCexplorer and CIRI, have been designed based on the identification of special junction-spanning sequences from transcriptome deep-sequencing samples (13). Many circRNAs derived from exonic, intergenic and intronic sequences have been identified using these approaches. At present, there are several circRNA databases that summarize and integrate the information obtained from large-scale circRNA identification studies, and among them, CIRCpedia and circBase are representative examples. CIRCpedia is an integrative database that has now been updated to CIRCpedia v2; this database contains comprehensive information, including circRNA annotations, expression patterns and conservation across six different species (14). For each of these species, CIRCpedia v2 enables users to search, browse and download circRNA sequences and details of alternative back-splicing events along with information about their expression patterns in various cell types and tissues, including disease samples (14). Another database, CircBase, contains the circRNAs identified in humans, mice, fruit flies and nematodes. Using CircBase, researchers can directly download the relevant sequences and annotation information of circRNAs of interest (15).

In recent years, hundreds of circRNAs have been confirmed to be dysregulated in specific malignant tumors, including gastric cancer (GC), hepatocellular carcinoma (HCC), breast cancer, osteosarcoma (OS) and tumors of the central nervous system (16-18). These previous studies indicated that circRNAs may potentially play powerful roles in tumor pathogenesis (19-21). Importantly, some circRNAs can be detected in saliva, plasma and exosomes, suggesting that they might be useful as biomarkers for tumor diagnosis (22-26). In the present review, the current knowledge on the biogenesis, classification and functions of circRNAs are briefly introduced, and their potential roles in malignant tumors are described.
The prospects of using circRNAs in clinical applications are also discussed.

\section{Biogenesis and classification of circRNAs}

In the canonical splicing process, introns are removed from precursor mRNAs (pre-mRNAs) and the exons are then covalently connected, thus forming a linear RNA molecule that can encode proteins (Fig. 1A) (27). However, circRNAs usually result from a noncanonical form of alternative splicing that connects the 5'caps and 3'polyadenylated tails (27-29). Initially, the circRNAs detected in human cells mainly originated from exons. However, via more in-depth studies, researchers found that various gene structures can generate a variety of circRNAs. The circRNA splicing mechanisms are much more complex than previously thought (30). Furthermore, alternative back-splicing selectively acts on different splice donors at the downstream 5'end or splice acceptors at the upstream 3'end; thus, the same region of a gene can also produce different types of circRNAs (30). In summary, the complexity in the variety of circRNAs is further expanded via alternative back-splicing.

Although there are many diverse forms of circular RNAs, they are mainly divided into three categories, according to their constituent sections (31). The first type is known as exonic circRNAs (ecRNAs), and they originate from single or several exons in pre-mRNAs. The second type, intron circRNAs (ciRNAs), only contain introns. Finally, exon-intron circRNAs (EIciRNAs) are a recently discovered type of circRNA that contain both exons and introns $(31,32)$. Among the three types, both ciRNAs andEIciRNAs are mainly localized to the nucleus, while ecRNAs are mainly present in the cytoplasm $(4,8,32,33)$. Although the mechanism of circRNA biogenesis is not yet fully understood, previous studies have revealed that back-splicing is catalyzed by the canonical spliceosome machinery, while both protein factors and cis complementary sequences, ALU repeats in particular, can modulate the process (4). In 2013, Jeck et al (4) first proposed two models to explain the formation of circRNAs: Lariat-driven circularization (exon skipping) and intron-pairing driven circularization (direct back-splicing). In the first model (Fig. 1B), an exon-containing lariat is created from pre-mRNA, and the introns contained in the lariat are then removed, thus generating an exonic circRNA (ecircRNA) (4,33). In the second model (Fig. 1C), ALU complementarity-dependent base-pairing supports the connection of a downstream splice donor pair having a non-spliced upstream splice acceptor, and the contributing RNA are covalently closed (33). The introns are then removed to form ecircRNAs (32), and, under some circumstances, these introns can be retained as EIciRNAs (33). Furthermore, accumulating evidence has verified that the second model might occur more frequently than the first (34). It has also been demonstrated that some RNA binding proteins (RBPs), such as protein muscleblind (MBL, a protein encoded by the mbl gene) and protein quaking (QKI, a splicing factor that promotes myelination and oligodendrocyte differentiation), can serve as bridges between the flanking introns to support the connection of the splice donor and acceptor to form intronic-paired RNAs, thereby facilitating circRNA production (Fig. 1D) $(35,36)$. Another model for ciRNA biogenesis (Fig. 1E) was reported by Zhang et al (37). They proposed that 


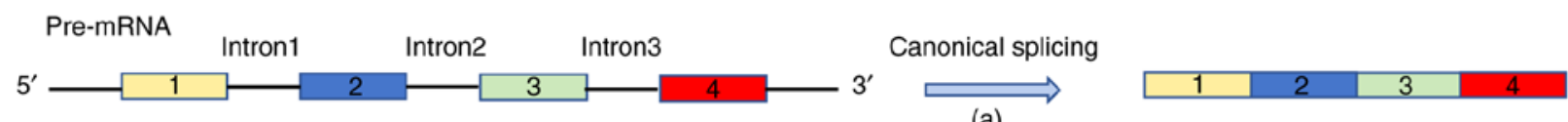

(a)

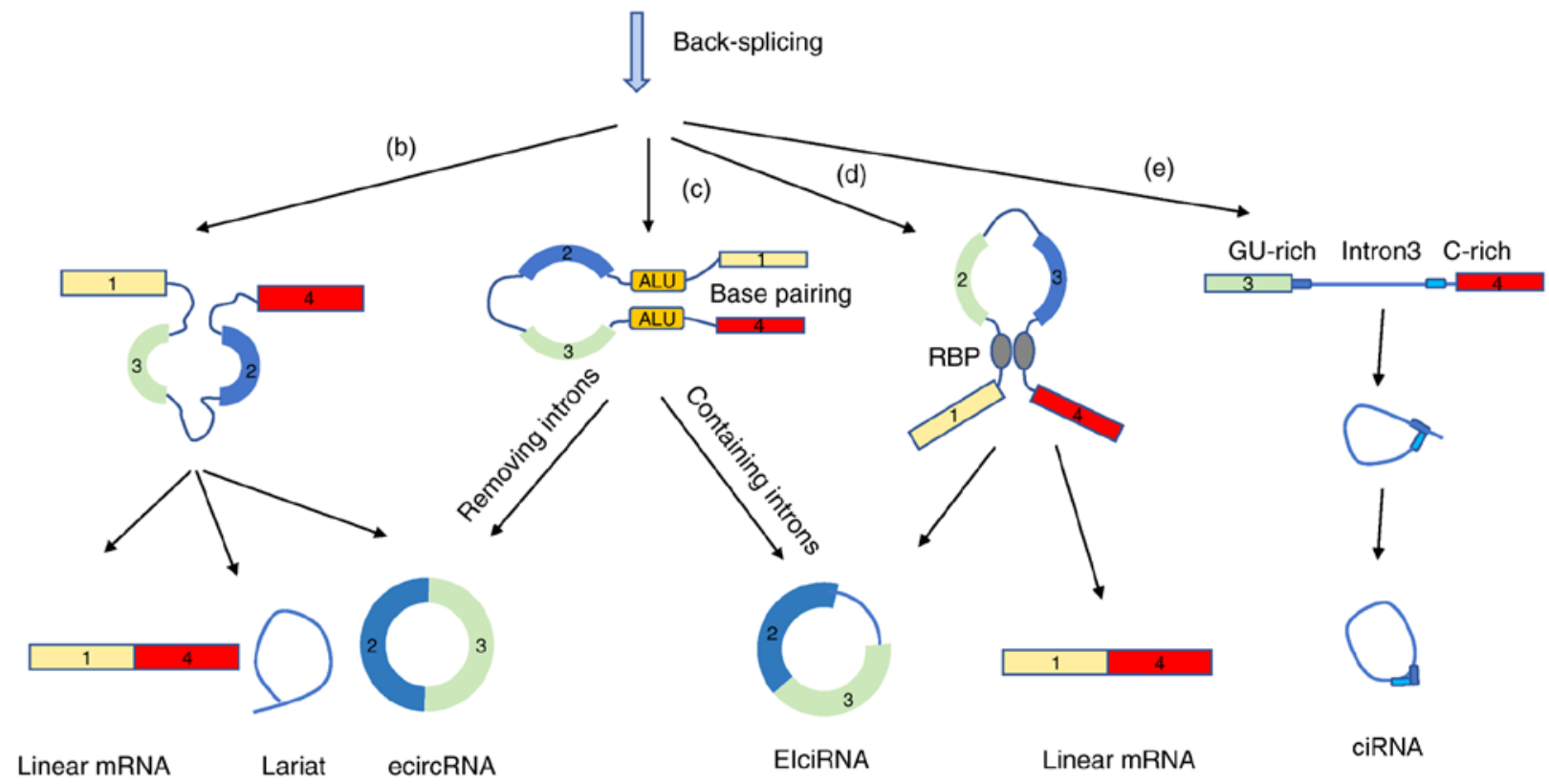

Figure 1. Biogenesis of linear RNA and different types of circRNAs. (A) A linear RNA molecule is derived from pre-mRNA via the canonical splicing process. (B) Lariat-driven circularization (exon skipping): An exon-containing lariat is first formed from a pre-mRNA. Next, intron removal results in the formation of an ecircRNA, intron lariat, and linear mRNA. (C) Intron pairing-driven circularization: The introns flanking inverted repeats or ALU elements promote the circularization of the exon/exons via direct base pairing. Next, EIciRNAs or ecircRNAs are formed via intron retention of removal. (D) RBP binding: RBPs act as bridges to connect specific sequence motifs of the flanking introns of linear pre-mRNAs, thus promoting back-splicing and exon circularization. (E) ciRNA biogenesis: Some lariats removed from pre-mRNAs via the canonical splicing machinery can form ciRNA molecules. This process depends on a consensus motif containing a 7-nt GU-rich element close to the 5'splice site and an 11-nt C-rich element close to the branchpoint site. circRNA, circular RNA; pre-mRNA, precursor mRNA; ecircRNA, exonic circRNA; EIciRNAs, exon-intron circRNAs; RBP, RNA binding protein.

the process of generating ciRNAs depends on a consensus motif containing a 7-nt GU-rich element near the 5'splice site and an 11-nt C-rich element near close to the branchpoint site (37). Furthermore, Wang et al (38) revealed that ciRNAs can also be formed by partial degradation of lariat RNAs. Currently, nuclear factor 90 , encoded by the interleukin enhancer binding factor 3 gene, and its 110 isoform NF110 have been confirmed to promote circRNA generation in the nucleus by stabilizing intronic RNA pairs (35). Another RBP, RNA-binding protein FUS, has particularly interesting functions, as several FUS genetic mutations may be associated with amyotrophic lateral sclerosis (ALS) $(39,40)$. Errichelli et al $(41)$ revealed that FUS promotes circRNA production via involvement in the control of back-splicing events, which has been linked to ALS pathology. Notably, the use of Csy4 (a cas-9 homolog in the CRISPR protein family) to activate RNA circularization could improve the efficiency and specificity of circRNA expression, and reduce the levels of the corresponding linear RNA by-products (42).

\section{Biological functions of circRNAs}

miRNA sponges. miRNAs are 23 -nt ncRNAs that have essential functions in genetic regulatory networks and are closely associated with many biological processes, such as cell apoptosis, proliferation, immune responses and tumorigenesis (43-45). In general, miRNAs can inhibit the function of target mRNAs by binding to their 3'untranslated regions (46). As a new classification of competing endogenous RNA (ceRNA), circRNAs with miRNA response element can affect the stability or the translation of target mRNAs by competitively binding to the same miRNA (Fig. 2A) (46). Furthermore, circRNAs are more effective at miRNA binding than other ceRNAs because of their abundance and stability (31). The earliest experimental support for the sponge function of circRNAs was provided by work on circular RNA sponge for miR-7 (ciRS-7), which originates from an antisense long ncRNA; in fact, ciRS-7 contains >70 miRNA-binding sites for miR-7 itself and has functions that affect brain development (47). Therefore, miR-7 can be inhibited from binding to other target sites by binding to ciRS-7. As a result, the levels of miR-7 target mRNAs can be increased $(7,8)$. Additionally, circ-sry derived from the sex-determining region $\mathrm{Y}$ that harbors many miR-138 binding sites, functions as an miR-138 sponge $(7,48)$. Chen et al $(49)$ determined via RT-qPCR that the expression of circ-anaphase-promoting complex subunit 7 (ANAPC7) was significantly upregulated in acute myeloid leukemia (AML) (49). Bioinformatics analysis predicted that circ-ANAPC7 could bind to miR-181 family members, which are closely associated with AML pathogenesis and prognosis (49).

With advances in circRNA research, increasing evidence suggested that the miRNA sponge activity may be a general function of circRNAs and, more importantly, that 


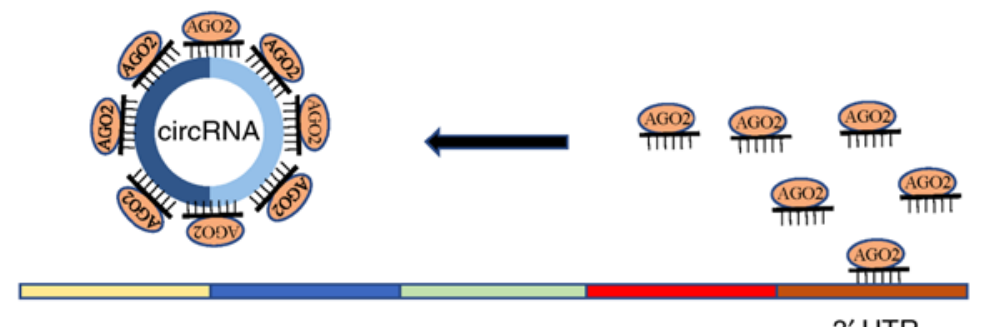

(a)

mRNA

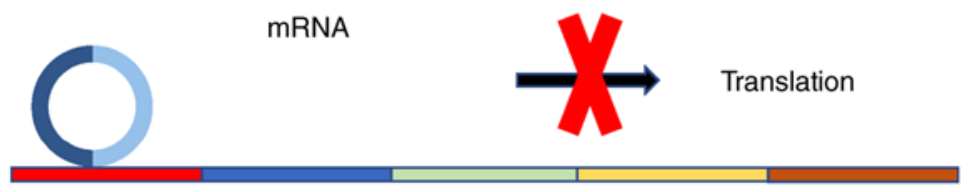

(e)

Translation start site

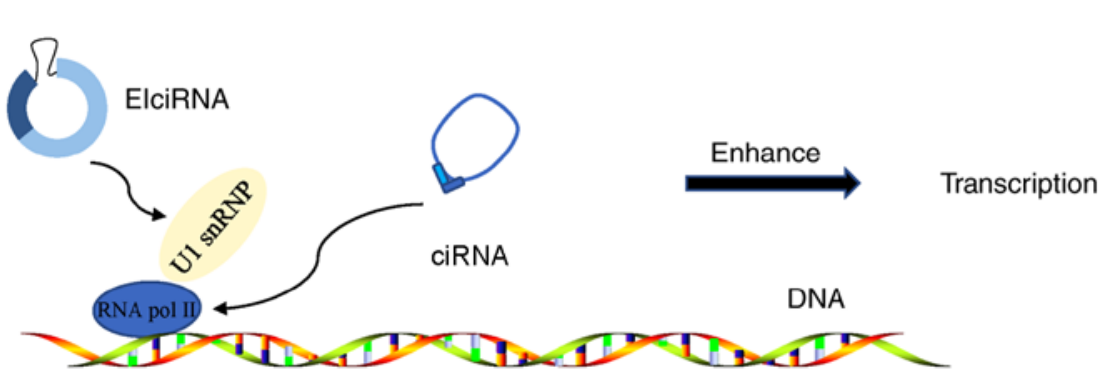

(d)

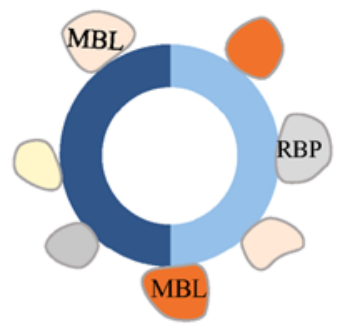

(b)

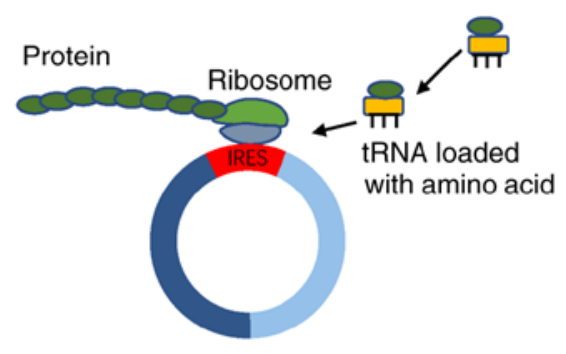

(c)

Figure 2. Functions of circRNAs. (A) circRNAs act as miRNA sponges. (B) circRNAs bind to proteins, such as RBP and MBL. (C) circRNAs act as translation templates. (D) circRNAs regulate transcription. (E) circRNAs regulate protein expression. circRNA, circular RNA; miRNA, microRNA; RBP, RNA binding protein; MBL, protein muscleblind; AGO2, argonaute-2; UTR, untranslated region; EIcirRNA, exon-intron circRNA; snRNP, small nuclear ribonucleoproteins; ciRNA, intron circRNA; IRES, internal ribosomal entry site; tRNA, transfer RNA.

circRNA/miRNA/mRNA coexpression networks have widespread involvement in the development of diverse types of malignant tumors. It should also be noted that the binding of miRNAs to circRNAs might not always lead to the suppression of the target miRNAs. In some situations, circRNAs can temporarily store or transport miRNAs, thus regulating the expression levels of the miRNA target mRNAs. For instance, ciRS-7 contains a near perfect complementary region to the miR-671 molecule (50). A complex of ciRS-7 and miR-671 can trigger the cleavage of ciRS-7 by protein argonaute-2 (AGO2) in an miR-671-dependent manner, thus releasing the bond to miR-7 (50).

Protein binding. In addition to serving as microRNA sponges, circRNAs can also directly bind proteins to participate in various biological processes (Fig. 2B). A typical example is circMbl, which is derived from the human muscleblind like splicing regulator 1 gene or the mbl gene in flies. circMbl has been reported to contain many conserved MBL binding sites $(28,51)$. Strong and specific binding of excess MBL to circMbl can regulate the production balance of MBL and circMbl $(28,51)$. Another circRNA, circ-Foxo3, which is derived from the forkhead box 03 gene, can bind to some proteins involved in the cell cycle, thus affecting cell cycle progression $(52,53)$. Du et al $(53)$ found that circ-Foxo3 is highly expressed in non-cancerous cells and is associated with cell cycle progression. Through a series of experiments, they revealed that circ-Foxo3 can interact with the cell cycle proteins cyclin-dependent kinase 2 (CDK2) and cyclin-dependent kinase inhibitor 1 (p21) to form a circ-Foxo3-p21-CDK2 ternary complex, thereby inhibiting progression from $G_{1}$ to $S$ phase (53). However, some circRNAs influence posttranslational regulation by interacting with RBPs, such as argonaute, QKI and Hu-antigen R (HuR) (8,34,50). For example, ciRS-7 interacts strongly with miR-7 and AGO2 proteins, both of which can reduce the expression and activity of mature miRNAs, and the specific miR-7-AGO2 interaction can inhibit miR-7 activity (8). In addition, CircPABPN1, at high levels, binds to HuR, an extensively studied RBP that influences gene expression programs by associating with hundreds of coding and non-coding linear RNAs, thus suppressing its binding to its cognate mRNA (polyadenylate-binding nuclear protein 1 mRNA) (54).

circRNA translation. In total, $>80 \%$ of the identified circRNAs originate from exons (ecRNAs) and are mainly located in the cytoplasm $(4,7,8)$. As is well known, exons can be translated into proteins during protein biosynthesis (55). Therefore, whether circRNAs have the potential to encode polypeptides or proteins requires investigation. Indeed, convincing evidence has demonstrated that some circRNAs containing open reading frames (ORFs) and internal ribosomal entry site (IRES) elements encode proteins via a cap-independent mechanism (Fig. 2C) (55). One of the first studies supporting the notion that natural circRNA can be translated into a protein investigated hepatitis $\delta$ virus (HDV). Wang and Wang (56) found a 
circular single stranded RNA with an OFR and a TGA stop codon presented in HDV that could behave as a translational template to express a single viral protein of 122 amino acids, in a non-canonical manner. Subsequently, scientists engineered an IRES into synthetic circRNAs and demonstrated that these IRES-containing circRNAs can be translated into green fluorescent proteins (56). For example, previous study have reported that eukaryotic ribosomes can initiate translation on circRNAs containing an IRES (57). Recent studies have also suggested that specific endogenous eukaryotic circRNAs can be translated into proteins $(41,58,59)$. For example, Circ-ZNF609, a circRNA derived from the circularization of the second exon of the zinc finger protein 609 (ZNF609) gene, is involved in controlling myoblast proliferation. Researchers identified a $753-n t$ ORF via analysis of the circZNF609 sequence $(58,59)$. Importantly, this ORF, which spans from a start codon to a stop codon, encodes a novel protein whose expression is driven by an IRES $(58,59)$. Another previous study by Yang et al (59) showed that circ-FBXW7 encodes a novel 185-amino acid protein, which they termed FBXW7-185aa. Downregulation of FBXW7-185aa promoted malignancy phenotypes, while upregulation of FBXW7-185aa inhibited cancer cell proliferation (59). Recently, Zhao et al (60) developed a new powerful bioinformatics tool, IRESfinder, that can be used to identify and analyze IRES components. Furthermore, Yang et al (61) discovered that some 'negative control' sequences (without IRES elements) can also be translated. The authors examined the sequences near the translation start site to try to explain this strange phenomenon (61). Their results showed that all of the relevant 'negative control' sequences contained an RRACH fragment $(\mathrm{R}=\mathrm{G}$ or $\mathrm{A} ; \mathrm{H}=\mathrm{A}, \mathrm{C}$ or $\mathrm{U})$ near the start codon, which resembles the consensus motif for N6-methyladenosine (m6A) modification (the RRACH motif) (61). Further experiments showed that m6A motifs were enriched in many circRNAs and that they can drive protein translation in human cells (61).

Transcription and splicing regulators. Unlike ecRNAs, circRNAs containing intron sequences (such as ciRNA EIciRNA) are predominantly localized to the nucleus, where they may interfere with the transcription of parental genes or regulate the splicing process (Fig. 2D). It has been reported that ciRNAs can interact with eukaryotic Pol II to participate in the efficient transcription of their parental genes (37). Ci-ankrd52, which is derived from the second intron of ankyrin repeat domain 52 (ANKRD52) and is enriched in the nucleus, can promote the transcription of the ANKRD52 gene by interacting with the Pol II elongation complex (37). Likewise, U1 snRNA is associated with some factors that participate in transcription initiation, elongation or termination (62). The interaction between EIciRNAs and U1 snRNA can also play a transcriptional regulatory role in the cell nucleus $(31,62,63)$. EIciRNAs containing U1 snRNA-binding sites in the retained introns, such as EIciPAIP2 and EIciEIF3J, have been confirmed to bind to U1 snRNA to enhance the transcription of EIF3J and PAIP2 in cis (32). Use of a U1 snRNA-specific antisense morpholino to knockdown U1 snRNA expression abolishes the regulatory effect of EIciRNA on the transcription of its parental gene (63).

Some circRNAs can act as splicing regulators to influence the process of linear splicing. As mentioned earlier, circMbl can regulate the production balance of MBL and circMbl by binding to surplus MBL. In addition, there are many MBL binding sites in the intronic sequences flanking the second exon of mbl. Some MBL isoforms can interact with the flanking introns to promote exon circularization. Therefore, circMbl might function in gene regulation by competing with canonical pre-mRNA splicing (28). In fact, for the majority of host genes, there is a negative interaction between circular and linear splicing. The formation of circRNAs can compete with canonical pre-mRNA splicing to reduce the levels of linear mRNAs. In this way, the levels of the proteins encoded by the linear mRNAs can be reduced $(28,64)$. Notably, some circRNAs containing translation start sites can directly sequester target mRNAs to regulate the translation of the remaining truncated linear mRNA (Fig. 2E) (34).

Other functions. Oncogenic 'fusion proteins' resulting from chromosomal translocations are involved in tumorigenesis (65). Interestingly, recent studies have also revealed that chromosomal translocations can lead to the production of fusion circRNAs (f-circRNA) that exert unique biological functions in tumor cells. For instance, protein PML $(\mathrm{PML}) /$ retinoic acid receptor $\alpha(\mathrm{RAR} \alpha)$ translocation is most recurrent in acute promyelocytic leukemia (APL), and aberrant MLL/AF9 translocation usually occurs in AML (66). Guarnerio et al (66) identified two types of f-circRNA that correspond to different exons associated with PML/RAR $\alpha$ and MLL/AF9 fusion genes: One is termed f-circPR and the other is termed f-circM9_1 (66). Both of these f-circRNAs can activate the PI3K and MAPK signaling transduction pathways, thus exerting pro-proliferative and proto-oncogenic effects. Furthermore, these f-circRNAs may also protect tumor cells from drug-induced apoptosis (66). Recently, Tan et al (67) reported a fusion circRNA(F-circEA) that originates from the EML4-ALK fusion gene and is mainly located in the cytoplasm. The results of Transwell assays and wound healing experiments showed that F-circEA can promote cell migration and invasion. Most importantly, F-circEA is not only present in non-small cell lung cancer (NSCLC) tissues but also specifically present in the plasma of patients carrying the EML4-ALK translocation (67). However, no F-circEA was detected in the patients lacking the fusion gene. These findings suggested that F-circEA could be a novel biomarker to monitor the EML4-ALK fusion gene status in NSCLC and to guide EML4-ALK-targeted NSCLC therapy (67).

\section{4. circRNAs in malignant tumors}

Many circRNAs have been demonstrated to be involved in cancer-related processes, including epithelial-mesenchymal transition (EMT), tumor immunity and exosome-mediated cell communication, in various tumor types. As one of the most important signaling pathways, EMT plays a crucial role in tumor metastasis. In GC, inhibiting circRNA_0023642 expression promoted E-cadherin expression, but decreased the expression of $\mathrm{N}$-cadherin, vimentin and snail, suggesting that circRNA_0023642 could modulate the activity of the EMT signaling pathway (68). In another previous study, Zeng et al (69) revealed that circANKS1B can promote breast cancer metastasis by sponging miR-148a-3p and miR-152-3p 
to increase the expression of upstream transcription factor 1, which could transcriptionally upregulate TGF- $\beta 1$ expression to promote EMT via activation of TGF- $\beta 1 /$ Smad signaling (69). An increasing number of previous studies have shown that circRNAs may play complicated and significant roles in tumor immunity by interacting with miRNAs or proteins. As previously reported, hsa_circ_0020397 was upregulated in colorectal cancer and antagonizes miR-138 to subsequently enhance the expression of miR-138 targets, including programmed cell death 1 ligand 1 (PD-L1) and telomerase reverse transcriptase (70). Upon PD-L1 upregulation, it can interact with programmed cell death protein 1 specifically on tumor cell surfaces to exhaust the immunocytes and induce cancer immune escape. CircFoxo3 can form complexes with E3 ubiquitin-protein ligase Mdm2, thus causing degradation of $\mathrm{p} 53$, a key protein involved in immune responses during tumorigenesis $(53,71)$. circRNAs can also participate in exosome-mediated cell communication. CiRS-133, which is significantly upregulated in GC, can be delivered into preadipocytes via exosomes, where it plays a crucial role in aggravating tumor cachexia (72). In 2018, Zhang et al (69) showed that adipose-derived exosomes mediate the delivery of circ-DB (a circRNA involved deubiquitylation), thus promoting $\mathrm{HCC}$ tumorigenesis via inhibition of miR-34a expression and activation of the ubiquitin carboxyl-terminal hydrolase 7/Cyclin A2 signaling pathway (18). A recent study by Wang et al (73) identified a number of dysregulated circRNAs in exosomes from the serum of patients with breast cancer. Previous studies of circRNAs in several common malignancies are discussed and detailed information is presented in Table I.

In $G C . \mathrm{GC}$ is a malignant tumor originating from the gastric mucosal epithelium and has the third highest cancer-related mortality rate worldwide (16). Sun et al (74) collected and screened a total of 5 GC tissue and adjacent non-tumorous tissue samples via a human circRNA microarray. By analyzing the t-test data from the screened patient samples, 713 candidate circRNAs were identified, among which 191 were upregulated and 522 were downregulated. RT-qPCR and bioinformatics-based predictions were used to analyze the 10 most significantly up- and downregulated circRNAs, and the results showed that the expression level of circPVRL3 was significantly decreased, suggesting that it might have an important biological function. Finally, a previous study revealed a molecular mechanism through which circPVRL3 could act as a sponge for $9 \mathrm{miRNAs}$ to regulate the proliferation and migration of GC (74). Another circRNA, hsa_circ_0000096, which is frequently downregulated in GC tissue, might suppress GC cell growth and migration by interfering with the expression of cell cycle-related proteins (e.g., cyclin D1 and CDK6) and migration-related proteins [e.g., matrix metalloproteinase (MMP)-2 and MMP-9] (75). Inhibition of hsa_circ_0000096 expression suppressed GC cell proliferation (75). In addition, the level of hsa_circ_0000096 in GC was significantly correlated with sex, invasion and TNM stage, suggesting that hsa_circ_0000096 may be a potential biomarker for GC diagnosis (75).

In $\mathrm{HCC}$. $\mathrm{HCC}$ is the most common primary malignancy of the liver (76). An increasing number of studies have demonstrated that aberrant circRNA expression can affect HCC development. For instance, circC3P1 is a circRNA derived from exons of the complement component 3 precursor pseudogene (C3P1), and it contains miR-4641 binding sites (77). Zhong et al (77) reported that circC3P1 expression was typically downregulated in HCC tissue compared with adjacent normal tissue. It was identified that the circC3P1 level was negatively correlated with tumor size, TNM stage and vascular invasion in HCC (77). Overexpression of circC3P1 was shown to be an inhibitory factor for HCC growth and metastasis (77). In another study, Jiang et al (78) identified a circRNA named hsa_circ_0000673 that was upregulated in HCC patient samples. High hsa_circ_0000673 expression was correlated with HCC progression, and it was shown to promote HCC proliferation and invasion by regulating the miR-767-3p/SET axis (78).

In breast cancer. Breast cancer is a malignant tumor that originates from the glandular epithelium of the breast and mainly occurs in women (79). Tang et al (80) used circRNA microarray assays to detect the circRNA expression profiles in breast cancer tissue samples, and they found that 1,705 circRNAs were abnormally expressed. Further screening clearly showed that among those circRNAs, 15 circRNAs were upregulated and 16 were downregulated (fold changes $>4.0$ and P-values <0.05) (80). More recently, a circular RNA named circ-Dnmt1 was shown to enhance the proliferation and survival of breast cancer cells by activating autophagy (81). Overexpressed circ-Dnmt1 can bind to p53 and AU-rich element RNA-binding protein 1 to promote their nuclear translocation, thereby inducing cellular autophagy and increasing DNA (cytosine-5)-methyltransferase 1 expression (81).

In lung cancer. Lung cancer is an important malignant tumor with high morbidity and mortality rates worldwide. cir-ITCH is a circRNA that plays an inhibitory role in lung cancer. Wan et al (82) found that cir-ITCH expression was significantly decreased in lung cancer tissue samples and that its expression level was associated with age (but not sex), tumor type and TNM stage. Aberrant cir-ITCH expression can suppress the activation of the Wnt/ $\beta$-catenin pathway, as cir-ITCH can act as a sponge for miR-7 and miR-214, thus leading to suppression of lung cancer progression (82). Another regulatory network involving circMAN2B2/miR-1275/forkhead box protein $\mathrm{K} 1$ (FOXK1) has also been reported in lung cancer. There is a negative correlation between circMAN2B2 and miR-1275 expression levels in lung cancer tissue. FOXK1 is a direct target of miR-1275, and overexpression of FOXK1 could reverse the inhibition of cell proliferation caused by circMAN2B2 knockdown. Experiments demonstrated that circMAN2B2 could promote lung cancer cell proliferation and invasion by modulating miR-1275-FOXK1 signaling (83). In another previous study, Zhang et al (84) studied 46 patients (11 females and 35 males) and ultimately focused on a circRNA termed hsa_circ_0014130 (84). This circRNA was upregulated in NSCLC tissue samples compared with paired adjacent lung tissue samples, and was significantly associated with TNM stage and lymphatic metastasis, suggesting that hsa_circ_0014130 may be a potential biomarker for NSCLC (84). 


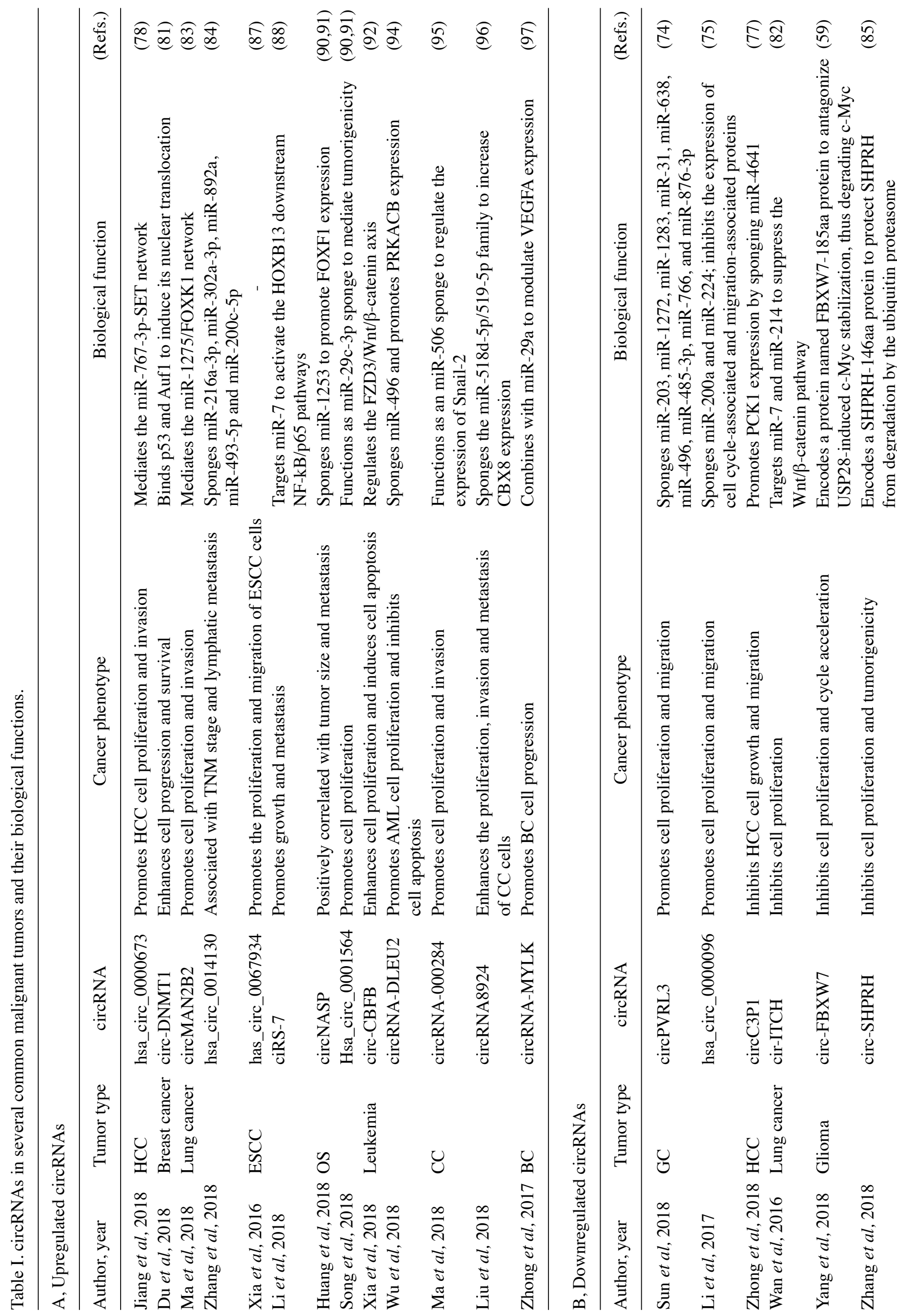


In In glioma. Yang et al (59) analyzed RNA-seq data from 10 glioblastoma and adjacent nontumorous tissue samples and identified $\sim 31,000$ circRNA candidates. Interestingly, circ-FBXW7, a circRNA derived from the F-box and WD repeat domain containing 7 (FBXW7) gene, which is a well-characterized tumor suppressive E3 ligase, was found to encode a novel 21-kDa protein termed FBXW7-185aa. FBXW7-185aa can antagonize ubiquitin carboxyl-terminal hydrolase 28-induced c-Myc stabilization to reduce the half-life of c-Myc, inhibiting cell proliferation (59). Similarly, Zhang et al (85) identified a protein encoded by circ-SHPRH that is downregulated in glioblastoma and acts as a tumor suppressor in glioblastoma. Jin et al (86) found that the expression of circHIPK 3 was significantly increased in glioma tissues. circHIPK3 can mediate tumorigenesis as a miR-654 sponge to promote insulin-like growth factor 2 mRNA-binding protein 3 (IGF2BP3) expression, and IGF2BP3 has been reported as an oncogene in many cancer types.

In esophageal squamous cell carcinoma (ESCC). ESCC is one of the most prevalent cancer types worldwide with a high mortality rate. Using RT-qPCR, Xia et al (87). found that the expression of has_circ_0067934 was markedly upregulated in ESCC. Hsa_circ_0067934 expression was significantly correlated with TNM stage and T stage. Moreover, the proliferation and migration of ESCC cells can be inhibited by knocking down hsa_circ_0067934 expression. These previous results suggested that hsa_circ_0067934 might represent a novel potential biomarker for ESCC (87). Another circRNA, ciRS-7, participates in the pathogenesis of diverse tumors (84). Li et al (88) found that ciRS-7 expression is also upregulated in ESCC. They further characterized a molecular mechanism through which ciRS-7 could as an miR-7 sponge to activate homeobox protein Hox-B13 downstream of the NF-kB/p65 pathway, thereby promoting ESCC growth and metastasis.

In OS. The involvement of hsa_circ_0002052 in impairing OS progression has beenrecently demonstrated.Hsa_circ_0002052 was significantly downregulated in OS tissue and cell lines compared with normal cell lines and adjacent normal tissues, respectively, and its overexpression was negatively correlated with OS cell proliferation, migration and invasion (17). A previous study revealed that hsa_circ_0002052 can modulate the miR-1205/adenomatous polyposis coli protein 2 axis to suppress the activation of the Wnt/ $\beta$-catenin pathway, leading to the inhibition of OS cell growth (17). Similarly, circHIPK3 was also poorly expressed in OS tissue, and its expression level was correlated with shorter overall survival time and poor prognosis (89). In contrast, Hsa_circ_0001564 and circNASP are upregulated in OS tissue, and knockdown of their expression markedly suppressed OS cell proliferation $(90,91)$.

In leukemia. Xia et al (92) analyzed RNA-seq data from 47 patients with chronic lymphocytic leukemia (CLL) and discovered that circ-CBFB expression was significantly upregulated in CLL tissue. circ-CBFB overexpression enhanced the proliferation and induced apoptosis of CLL cells by regulating the miR-607/frizzled-3/Wnt/ $\beta$-catenin axis (92). Li et al (93) discovered that numerous circRNAs are aberrantly expressed in APL samples compared with their levels in normal blood 
samples and other non-APL AML samples. Among these, circ-HIPK2, which is significantly downregulated in patients with APL, was shown to be a potential APL-associated biomarker (93). In another previous study by Wu et al (94), circRNA-DLEU2 was found to be associated with the inhibition and proliferation of AML cells.

In cervical cancer $(C C)$. circRNA-000284 is one of the upregulated circRNAs in cervical cancer (CC) cells. circRNA-000284 upregulation promotes $\mathrm{CC}$ cell proliferation and invasion (95). Likewise, circRNA8924 is also highly expressed in CC. In vitro experiments verified that circRNA8924 overexpression increases chromobox protein homology 8 expression by sponging miR-518d-5p/519-5p family members, thereby enhancing the proliferation, invasion and metastasis of $\mathrm{CC}$ cells (96).

In bladder cancer $(B C)$. In $\mathrm{BC}, 469$ circRNAs that were differentially expressed between bladder carcinomas and matched para-carcinomas tissue were identified via microarray analysis, among which 285 were upregulated and 184 were downregulated. Furthermore, 4,416 mRNAs were also significantly differentially expressed (including 2,472 upregulated and 1,944 downregulated) (97). Among all circRNAs, circRNA-MYLK is abnormally upregulated in bladder cancer tissue, and its expression level is positively correlated with vascular endothelial growth factor A (VEGFA) mRNA levels. An interaction between circRNA-MYLK, miR-29a and VEGFA has been demonstrated via luciferase reporter assays. circRNA-MYLK can bind to miR-29a to modulate VEGFA expression, thereby inducing EMT and activating the Ras/ERK signaling cascade (97). In another previous study, Yang et al (98) identified 14 upregulated and 42 downregulated circRNAs between cancerous samples and non-cancerous samples (98). Similar to lung cancer, the circ-ITCH expression level was also found to be downregulated in $\mathrm{BC}$ tissue. Furthermore, it was found that circ-ITCH could induce $\mathrm{G}_{1}$ cell cycle arrest by sponging miR-17 and miR-224, thus leading to the proliferation, invasion and migration of bladder cancer (99).

\section{Clinical applications of circRNAs}

As potential biomarkers. It is well known that early detection and prompt treatment are very important determinants in the prognoses of malignant tumors. Many tumor diagnostic markers and tools, including computerized tomographic scanning, magnetic resonance imaging and histopathology are already used in the clinical setting (31). However, some of these approaches are invasive and very expensive; thus, less expensive and minimally invasive or noninvasive approaches are preferred for tumor diagnosis. Dysregulation of circRNAs between tumor tissues and matched non-tumorous tissues is common, and such dysregulation is correlated with clinical pathological features, including tumor size and TNM stage (100). circRNAs are generally expressed in a tissue/developmental-stage-specific manner (64). Due to their loop structure, lacking terminal 5' and 3'ends, circRNAs are resistant to ribonucleases, such as exonuclease or RNase $\mathrm{R}$ (4). Therefore, they are more stable and have longer half-lives compared with linear mRNAs. Previous studies demonstrated that some circRNAs can be detected in saliva and plasma $(22,23)$. In addition, Li et al (101) first reported that circRNAs are abundant in human serum exosomes and that they are highly stable. By employing a xenograft mouse model, they demonstrated that tumor-derived exosomal circRNAs could enter circulation and then be measured for cancer detection $(64,101)$. Dou et al (22) found a global downregulation of circRNAs in colon cancer cell lines that depended only on their KRAS mutation status. Interestingly, circRNA molecules can be transferred to exosomes and can be more abundant in exosomes than in cells, suggesting that they may serve as promising cancer biomarkers. By analyzing RNA-seq samples, Alhasan et al (102) found that circRNAs are 17- to 188-fold enriched in human platelets relative to their levels in nucleated tissues (102). In addition, a recent study also showed that circRNAs are enriched in human hematopoietic cells and that their expression is widespread and cell-type specific (103). Importantly, the use of RT-qPCR and in situ hybridization for circRNA detection is more specific and sensitive compared with the detection of proteins via antigen-antibody reactions (31). Collectively, it was demonstrated that circRNAs have great potential for use as non-invasive diagnostic and prognostic biomarkers for malignant tumors.

As potential therapeutic targets and vectors. Based on all the information, it was determined that that circRNAs play different roles in diverse tumor types. Some circRNAs act as oncogenic factors to promote tumor development, while others act as tumor-suppressor factors to suppress tumor proliferation and growth. Moreover, that circRNAs function as miRNA sponges is currently the main mechanism for circRNAs participating in tumor progression. At present, some RNA-targeting drugs have been approved by The Food and Drug Administration. Therefore, the use of circRNAs as novel therapeutic targets or therapeutic vectors is an option for tumor treatment. There are different therapeutic strategies for circRNAs with different functions. In therapeutic strategies against carcinogenic circRNAs, a feasible approach is to deliver small interfering RNAs (siRNAs) that are perfectly complementary to the back-splice junction $(31,104)$. Another method is to use antisense oligonucleotides (ASOs) to bind the pre-mRNA of the target circRNAs via complementary base pairing $(31,104)$. Both methods can suppress the expression of oncogenic-circRNAs. In therapeutic strategies involving tumor suppressor circRNAs, a viable approach is to introduce exogenous genes to induce expression of the tumor-suppressor circRNA (104). However, this approach represents a new field and there are still many problems to be solved. For example, it is necessary to reduce the side effects associated with the siRNAs and to avoid the off-target gene silencing $(104,105)$. The designed ASOs also require stringent quality control to ensure that the target circRNAs can be specifically antagonized, thereby avoiding off-target effects due to cognate parental gene inhibition $(31,106)$. However, circRNAs are highly stable and have a superior ability to sponge proteins and miRNAs, suggesting that they may be a promising therapeutic vector. Introduction of specific circRNAs containing multiple binding sites for oncogenic miRNAs or proteins into tumor cells could restore normal regulatory networks, thus inhibiting tumor cell proliferation or inducing apoptosis. 


\section{Conclusions and perspectives}

The powerful functions and unique characters of circRNAs have attracted increasing attention from researchers worldwide. Due to a number of research advances, more information on circRNAs has been uncovered. Numerous dysregulated circRNAs have been identified in various malignant tumors. Meanwhile, new functions of circRNAs are continually providing new insight. For instance, circRNAs can act as ceRNAs to regulate and control tumor progression. They may also serve as targets for tumor treatment. Unusual for ncRNAs, some circRNAs can actually encode polypeptides or proteins. However, much remains to be learned about circRNAs, and some essential aspects of circRNA biology are not yet fully understood. At present, a systematic method for naming circRNAs is lacking. Additionally, how circRNAs are transported and degraded in vivo requires elucidation. The mechanisms that regulate the production of circRNAs are not fully understood. The exact molecular mechanisms underlying the involvement of circRNAs in tumor development also remain unclear. Further studies on these aspects will help to provide insight to better understand circRNA biology, thus further identifying potential clinical applications for circRNAs.

\section{Acknowledgements}

Not applicable.

\section{Funding}

The present study was supported by The National Natural Science Foundation of China (grant no. 81202115) and The Doctoral Innovation Fund of Shanghai Jiaotong University School of Medicine (grant no. BXJ201732).

\section{Availability of data and materials}

The datasets used and/or analyzed during the current study are available from the corresponding author on reasonable request.

\section{Authors' contributions}

WZ and JM conceived the study, performed the literature search and drafted the manuscript. MD and YW constructed the table. JP made the figures. XL and JZ revised the article and directed the review to be more focused. JM gave final approval for the article to be published. All authors read and approved the manuscript.

\section{Ethics approval and consent to participate}

Not applicable.

\section{Patient consent for publication}

Not applicable.

\section{Competing interests}

The authors declare that they have no competing interests.

\section{References}

1. Dong Y, Xu S, Liu J, Ponnusamy M, Zhao Y, Zhang Y, Wang Q, $\mathrm{Li} \mathrm{P}$ and Wang K: Non-coding RNA-linked epigenetic regulation in cardiac hypertrophy. Int J Biol Sci 14: 1133-1141, 2018.

2. Ma L, Bajic VB and Zhang Z: On the classification of long non-coding RNAs. RNA Biol 10: 925-933, 2013.

3. Esteller M: Non-coding RNAs in human disease. Nat Rev Genet 12: 861-874, 2011.

4. Jeck WR, Sorrentino JA, Wang K, Slevin MK, Burd CE, Liu J, Marzluff WF and Sharpless NE: Circular RNAs are abundant, conserved, and associated with ALU repeats. RNA 19: 141-157, 2013.

5. Abu N and Jamal R: Circular RNAs as promising biomarkers: A mini-review. Front Physiol 7: 355, 2016.

6. Sanger HL, Klotz G, Riesner D, Gross HJ and Kleinschmidt AK: Viroids are single-stranded covalently closed circular RNA molecules existing as highly base-paired rod-like structures. Proc Natl Acad Sci USA 73: 3852-3856, 1976.

7. Memczak S, Jens M, Elefsinioti A, Torti F, Krueger J, Rybak A, Maier L, Mackowiak SD, Gregersen LH, Munschauer M, et al: Circular RNAs are a large class of animal RNAs with regulatory potency. Nature 495: 333-338, 2013.

8. Salzman J, Gawad C, Wang PL, Lacayo N and Brown PO: Circular RNAs are the predominant transcript isoform from hundreds of human genes in diverse cell types. PLoS One 7: e30733, 2012.

9. Hansen TB, Jensen TI, Clausen BH, Bramsen JB, Finsen B, Damgaard CK and Kjems J: Natural RNA circles function as efficient microRNA sponges. Nature 495: 384-388, 2013.

10. Pasman Z, Been MD and Garcia-Blanco MA: Exon circularization in mammalian nuclear extracts. RNA 2: 603-610, 1996.

11. Lasda E and Parker R: Circular RNAs: Diversity of form and function. RNA 20: 1829-1842, 2014.

12. Cocquet J, Chong A, Zhang G and Veitia RA: Reverse transcriptase template switching and false alternative transcripts. Genomics 88: 127-131, 2006.

13. Lopez-Jimenez E, Rojas AM and Andrés-León E: RNA sequencing and prediction tools for circular RNAs analysis. Adv Exp Med Biol 1087: 17-33, 2018.

14. Dong R, Ma XK, Li GW and Yang L: CIRCpedia v2: An updated database for comprehensive circular RNA annotation and expression comparison. Genomics Proteomics Bioinformatics 16: 226-233, 2018.

15. Glazar P, Papavasileiou P and Rajewsky N: circBase: A database for circular RNAs. RNA 20: 1666-1670, 2014.

16. Figueiredo C, Camargo MC, Leite M, Fuentes-Pananá EM, Rabkin CS and Machado JC: Pathogenesis of gastric cancer: Genetics and molecular classification. Curr Top Microbiol Immunol 400: 277-304, 2017.

17. Wu Z, Shi $\mathrm{W}$ and Jiang C: Overexpressing circular RNA hsa circ_0002052 impairs osteosarcoma progression via inhibiting $\mathrm{Wnt} / \beta$-catenin pathway by regulating $\mathrm{miR}-1205 / \mathrm{APC} 2$ axis. Biochem Biophys Res Commun 502: 465-471, 2018.

18. Zhang H, Deng T, Ge S, Liu Y, Bai M, Zhu K, Fan Q, Li J, Ning T, Tian F, et al: Exosome circRNA secreted from adipocytes promotes the growth of hepatocellular carcinoma by targeting deubiquitination-related USP7. Oncogene 38: 2844-2859, 2019.

19. Rong D, Tang W, Li Z, Zhou J, Shi J, Wang H and Cao H: Novel insights into circular RNAs in clinical application of carcinomas. Onco Targets Ther 10: 2183-2188, 2017.

20. Shi Y, Guo Z, Fang N, Jiang W, Fan Y, He Y, Ma Z and Chen Y: hsa_circ_0006168 sponges miR-100 and regulates mTOR to promote the proliferation, migration and invasion of esophageal squamous cell carcinoma. Biomed Pharmacother 117: 109151, 2019.

21. Lu Q, Liu T, Feng H, Yang R, Zhao X, Chen W, Jiang B, Qin H, Guo X, Liu M, et al: Circular RNA circSLC8A1 acts as a sponge of $\mathrm{miR}-130 \mathrm{~b} / \mathrm{miR}-494$ in suppressing bladder cancer progression via regulating PTEN. Mol Cancer 18: 111, 2019.

22. Dou Y, Cha DJ, Franklin JL, Higginbotham JN, Jeppesen DK, Weaver AM, Prasad N, Levy S, Coffey RJ, Patton JG and Zhang B: Circular RNAs are down-regulated in KRAS mutant colon cancer cells and can be transferred to exosomes. Sci Rep 6: 37982, 2016.

23. Bahn JH, Zhang Q, Li F, Chan TM, Lin X, Kim Y, Wong DT and Xiao X: The landscape of microRNA, Piwi-interacting RNA, and circular RNA in human saliva. Clin Chem 61: 221-230, 2015. 
24. Yang C, Wei Y, Yu L and Xiao Y: Identification of altered circular RNA expression in serum exosomes from patients with papillary thyroid carcinoma by high-throughput sequencing. Med Sci Monit 25: 2785-2791, 2019.

25. Xiong S, Peng H, Ding X, Wang X, Wang L, Wu C, Wang S, $\mathrm{Xu} \mathrm{H}$ and Liu Y: Circular RNA expression profiling and the potential role of hsa_circ_0089172 in Hashimoto's thyroiditis via sponging miR125a-3p. Mol Ther Nucleic Acids 17: 38-48, 2019.

26. Guarnerio J, Zhang Y, Cheloni G, Panella R, Mae Katon J, Simpson M, Matsumoto A, Papa A, Loretelli C, Petri A, et al: Intragenic antagonistic roles of protein and circRNA in tumorigenesis. Cell Res: Jun 17, 2019 doi: 10.1038/s41422-019-0192-1 (Epub ahead of print).

27. Liang D and Wilusz JE: Short intronic repeat sequences facilitate circular RNA production. Genes Dev 28: 2233-2247, 2014

28. Ashwal-Fluss R, Meyer M, Pamudurti NR, Ivanov A, Bartok O, Hanan M, Evantal N, Memczak S, Rajewsky N and Kadener S: circRNA biogenesis competes with pre-mRNA splicing. Mol Cell 56: 55-66, 2014.

29. Muller $S$ and Appel B: In vitro circularization of RNA. RNA Biol 14: 1018-1027, 2017.

30. Zhang X, Dong R, Zhang Y,Zhang JL, Luo Z, Zhang J, Chen LL and Yang L: Diverse alternative back-splicing and alternative splicing landscape of circular RNAs. Genome Res 26: 1277-1287, 2016.

31. Zhang M and Xin Y: Circular RNAs: A new frontier for cancer diagnosis and therapy. J Hematol Oncol 11: 21, 2018.

32. Li Z, Huang C, Bao C, Chen L, Lin M, Wang X, Zhong G, Yu B, $\mathrm{Hu}$ W, Dai L, et al: Exon-intron circular RNAs regulate transcription in the nucleus. Nat Struct Mol Biol 22: 256-264, 2015.

33. Zhang XO, Wang HB, Zhang Y, Lu X, Chen LL and Yang L: Complementary sequence-mediated exon circularization. Cell 159: 134-147, 2014.

34. Jeck WR and Sharpless NE: Detecting and characterizing circular RNAs. Nat Biotechnol 32: 453-461, 2014.

35. Li X, Liu CX, Xue W, Zhang Y, Jiang S, Yin QF, Wei J, Yao RW Yang L and Chen LL: Coordinated circRNA biogenesis and function with NF90/NF110 in viral infection. Mol Cell 67: 214-227.e7, 2017.

36. Conn SJ, Pillman KA, Toubia J, Conn VM, Salmanidis M Phillips CA, Roslan S, Schreiber AW, Gregory PA and Goodall GJ: The RNA binding protein quaking regulates formation of circRNAs. Cell 160: 1125-1134, 2015.

37. Zhang Y, Zhang XO, Chen T, Xiang JF, Yin QF, Xing YH, Zhu S, Yang $\mathrm{L}$ and Chen LL: Circular intronic long noncoding RNAs. Mol Cell 51: 792-806, 2013.

38. Wang Z: Not just a sponge: New functions of circular RNAs discovered. Sci China Life Sci 58: 407-408, 2015.

39. Kwiatkowski TJ Jr, Bosco DA, Leclerc AL, Tamrazian E, Vanderburg CR, Russ C, Davis A, Gilchrist J, Kasarskis EJ, Munsat T, et al: Mutations in the FUS/TLS gene on chromosome 16 cause familial amyotrophic lateral sclerosis. Science 323 1205-1208, 2009.

40. Vance C, Rogelj B, Hortobágyi T, De Vos KJ, Nishimura AL, Sreedharan J, Hu X, Smith B, Ruddy D, Wright P, et al: Mutations in FUS, an RNA processing protein, cause familial amyotrophic lateral sclerosis type 6. Science 323: 1208-1211, 2009

41. Errichelli L, Dini Modigliani S, Laneve P, Colantoni A, Legnini I Capauto D, Rosa A, De Santis R, Scarfò R, Peruzzi G, et al: FUS affects circular RNA expression in murine embryonic stem cell-derived motor neurons. Nat Commun 8: 14741, 2017.

42. Borchardt EK, Meganck RM, Vincent HA, Ball CB, Ramos SBV, Moorman NJ, Marzluff WF and Asokan A: Inducing circular RNA formation using the CRISPR endoribonuclease Csy4. RNA 23: 619-627, 2017.

43. Liao Q, Wang B, Li X and Jiang G: miRNAs in acute myeloid leukemia. Oncotarget 8: 3666-3682, 2017.

44. Dragomir M, Mafra ACP, Dias SMG, Vasilescu C and Calin GA: Using microRNA networks to understand cancer. Int J Mol Sci 19: pii: E1871, 2018

45. Fumagalli MR, Zapperi S and La Porta CAM: Impact of the cross-talk between circular and messenger RNAs on cell regulation. J Theor Biol 454: 386-395, 2018

46. Zhong Y, Du Y, Yang X, Mo Y, Fan C, Xiong F, Ren D, Ye X, Li C, Wang Y, et al: Circular RNAs function as ceRNAs to regulate and control human cancer progression. Mol Cancer 17: 79, 2018

47. Piwecka M, Glazar P, Hernandez-Miranda LR, Memczak S, Wolf SA, Rybak-Wolf A, Filipchyk A, Klironomos F, Cerda Jara CA, Fenske P, et al: Loss of a mammalian circular RNA locus causes miRNA deregulation and affects brain function. Science 357: pii: eaam8526, 2017.
48. Capel B, Swain A, Nicolis S, Hacker A, Walter M, Koopman P, Goodfellow P and Lovell-Badge R: Circular transcripts of the testis-determining gene Sry in adult mouse testis. Cell 73: 1019-1030, 1993

49. Chen H, Liu T, Liu J, Feng Y, Wang B, Wang J, Bai J, Zhao W, Shen Y, Wang X, et al: Circ-ANAPC7 is upregulated in acute myeloid leukemia and appears to target the MiR-181 family. Cell Physiol Biochem 47: 1998-2007, 2018.

50. Hansen TB, Wiklund ED, Bramsen JB, Villadsen SB, Statham AL, Clark SJ and Kjems J: miRNA-dependent gene silencing involving Ago2-mediated cleavage of a circular antisense RNA. EMBO J 30: 4414-4422, 2011.

51. Du WW, Zhang C, Yang W, Yong T, Awan FM and Yang BB: Identifying and characterizing circRNA-protein interaction. Theranostics 7: 4183-4191, 2017.

52. Gartel A and Radhakrishnan SK: Lost in transcription: p21 repression, mechanisms, and consequences. Cancer Res 65: 3980-3985, 2005

53. Du WW, Yang W, Liu E, Yang Z, Dhaliwal P and Yang BB: Foxo3 circular RNA retards cell cycle progression via forming ternary complexes with p21 and CDK2. Nucleic Acids Res 44: 2846-2858, 2016

54. Abdelmohsen K, Panda A, Munk R, Grammatikakis I, Dudekula DB, De S, Kim J, Noh JH, Kim KM, Martindale JL and Gorospe M: Identification of HuR target circular RNAs uncovers suppression of PABPN1 translation by CircPABPN1. RNA Biol 14: 361-369, 2017.

55. Pamudurti NR, Bartok O, Jens M, Ashwal-Fluss R, Stottmeister C, Ruhe L, Hanan M, Wyler E, Perez-Hernandez D, Ramberger E, et al: Translation of CircRNAs. Mol Cell 66: 9-21. e7, 2017.

56. Wang Y and Wang Z: Efficient backsplicing produces translatable circular mRNAs. RNA 21: 172-179, 2015.

57. Chen CY and Science S: Initiation of protein synthesis by the eukaryotic translational apparatus on circular RNAs Science 268: 415-417, 1995.

58. Legnini I, Di Timoteo G, Rossi F, Morlando M, Briganti F, Sthandier O, Fatica A, Santini T, Andronache A, Wade M, et al: Circ-ZNF609 Is a Circular RNA that Can Be translated and functions in myogenesis. Mol Cell 66: 22-37.e9, 2017.

59. Yang Y, Gao X, Zhang M, Yan S, Sun C, Xiao F, Huang N, Yang X, Zhao K, Zhou H, et al: Novel Role of FBXW7 Circular RNA in repressing glioma tumorigenesis. J Natl Cancer Inst: 110, 2018 doi: $10.1093 /$ jnci/djx166.

60. Zhao J, Wu J, Xu T, Yang Q, He J and Song X: IRESfinder: Identifying RNA internal ribosome entry site in eukaryotic cell using framed k-mer features. J Genet Genomics 45: 403-406, 2018 .

61. Yang Y, Fan X, Mao M, Song X, Wu P, Zhang Y, Jin Y, Yang Y, Chen LL, Wang Y, et al: Extensive translation of circular RNAs driven by $\mathrm{N}^{6}$-methyladenosine. Cell Res 27: 626-641, 2017.

62. Kwek K, Murphy S, Furger A, Thomas B, O'Gorman W, Kimura H, Proudfoot NJ and Akoulitchev A: U1 snRNA associates with TFIIH and regulates transcriptional initiation. Nat Struct Biol 9: 800-805, 2002.

63. Huang C and Shan G: What happens at or after transcription: Insights into circRNA biogenesis and function. Transcription 6: 61-64, 2015

64. Qu S, Liu Z, Yang X, Zhou J, Yu H, Zhang R and Li H: The emerging functions and roles of circular RNAs in cancer. Cancer Lett 414: 301-309, 2018

65. Somervaille TC and Cleary ML: Grist for the MLL: How do MLL oncogenic fusion proteins generate leukemia stem cells? Int J Hematol 91: 735-741, 2010.

66. Guarnerio J, Bezzi M, Jeong JC, Paffenholz SV, Berry K, Naldini MM, Lo-Coco F, Tay Y, Beck AH and Pandolfi PP: Oncogenic role of Fusion-circRNAs derived from cancer-associated chromosomal translocations. Cell 165: 289-302, 2016.

67. Tan S, Gou Q, Pu W, Guo C, Yang Y, Wu K, Liu Y, Liu L, Wei YQ and Peng Y: Circular RNA F-circEA produced from EML4-ALK fusion gene as a novel liquid biopsy biomarker for non-small cell lung cancer. Cell Res 28: 693-695, 2018.

68. Zhou LH, Yang YC, Zhang RY, Wang P, Pang MH and Liang LQ: CircRNA 0023642 promotes migration and invasion of gastric cancer cells by regulating EMT. Eur Rev Med Pharmacol Sci 22: 2297-2303, 2018

69. Zeng K, He B, Yang BB, Xu T, Chen X, Xu M, Liu X, Sun H, Pan Y and Wang S: The pro-metastasis effect of circANKS1B in breast cancer. Mol Cancer 17: 160, 2018. 
70.Zhang XL, Xu LL and Wang F: Hsa_circ_0020397 regulates colorectal cancer cell viability, apoptosis and invasion by promoting the expression of the miR-138 targets TERT and PD-L1. Cell Biol Int 41: 1056-1064, 2017.

71. Huang Y, Yu P, Li W, Ren G, Roberts AI, Cao W, Zhang X, Su J, Chen X, Chen Q, et al: p53 regulates mesenchymal stem cell-mediated tumor suppression in a tumor microenvironment through immune modulation. Oncogene 33: 3830-3838, 2014

72.Zhang H, Zhu L, Bai M, Liu Y, Zhan Y, Deng T, Yang H, Sun W, Wang X, Zhu K, et al: Exosomal circRNA derived from gastric tumor promotes white adipose browning by targeting the miR-133/PRDM16 pathway. Int J Cancer 144: 2501-2515, 2019.

73. Wang J, Zhang Q, Zhou S, Xu H, Wang D, Feng J, Zhao J and Zhong S: Circular RNA expression in exosomes derived from breast cancer cells and patients. Epigenomics 11: 411-421, 2019.

74. Sun HD, Xu ZP, Sun ZQ, Zhu B, Wang Q, Zhou J, Jin H, Zhao A Tang WW and Cao XF: Down-regulation of circPVRL3 promotes the proliferation and migration of gastric cancer cells. Sci Rep 8: 10111, 2018

75. Li P, Chen H, Chen S, Mo X, Li T, Xiao B, Yu R and Guo J: Circular RNA 0000096 affects cell growth and migration in gastric cancer. Br J Cancer 116: 626-633, 2017.

76. Montagner A, Le Cam L and Guillou H: $\beta$-catenin oncogenic activation rewires fatty acid catabolism to fuel hepatocellular carcinoma. Gut 68: 183-185, 2019.

77. Zhong L, Wang Y, Cheng Y, Wang W, Lu B, Zhu L and Ma Y: Circular RNA circC3P1 suppresses hepatocellular carcinoma growth and metastasis through miR-4641/PCK1 pathway. Biochem Biophys Res Commun 499: 1044-1049, 2018.

78. Jiang W, Wen D, Gong L, Wang Y, Liu Z and Yin F: Circular RNA hsa_circ_0000673 promotes hepatocellular carcinoma malignance by decreasing miR-767-3p targeting SET. Biochem Biophys Res Commun 500: 211-216, 2018.

79. Seneviratne S, Lawrenson R, Scott N, Kim B, Shirley R and Campbell I: Breast cancer biology and ethnic disparities in breast cancer mortality in new zealand: A cohort study. PLoS One 10: e0123523, 2015.

80. Tang YY, Zhao P, Zou TN, Duan JJ, Zhi R, Yang SY, Yang DC and Wang XL: Circular RNA hsa_circ_0001982 promotes breast cancer cell carcinogenesis through decreasing miR-143. DNA Cell Biol 36: 901-908, 2017.

81. Du WW, Yang W, Li X, Awan FM, Yang Z, Fang L, Lyu J, Li F, Peng C, Krylov SN, et al: A circular RNA circ-DNMT1 enhances breast cancer progression by activating autophagy. Oncogene 37: 5829-5842,2018

82. Wan L, Zhang L, Fan K, Cheng ZX, Sun QC and Wang JJ: Circular RNA-ITCH suppresses lung cancer proliferation via inhibiting the Wnt/ $\beta$-catenin pathway. Biomed Res Int 2016 1579490, 2016.

83. Ma X, Yang X, Bao W, Li S, Liang S, Sun Y, Zhao Y, Wang J and Zhao C: Circular RNA circMAN2B2 facilitates lung cancer cell proliferation and invasion via miR-1275/FOXK1 axis. Biochem Biophys Res Commun 498: 1009-1015, 2018.

84.Zhang S, Zeng X, Ding T, Guo L, Li Y, Ou S and Yuan H: Microarray profile of circular RNAs identifies hsa_circ_0014130 as a new circular RNA biomarker in non-small cell lung cancer. Sci Rep 8: 2878, 2018

85. Zhang M, Huang N, Yang X, Luo J, Yan S, Xiao F, Chen W, Gao X, Zhao K, Zhou H, et al: A novel protein encoded by the circular form of the SHPRH gene suppresses glioma tumorigenesis. Oncogene 37: 1805-1814, 2018.

86. Jin P, Huang Y, Zhu P, Zou Y, Shao T and Wang O: CircRNA circHIPK3 serves as a prognostic marker to promote glioma progression by regulating miR-654/IGF2 BP3 signaling. Biochem Biophys Res Commun 503: 1570-1574, 2018.

87. Xia W, Qiu M, Chen R, Wang S, Leng X, Wang J, Xu Y, Hu J, Dong G, Xu PL and Yin R: Circular RNA has_circ_0067934 is upregulated in esophageal squamous cell carcinoma and promoted proliferation. Sci Rep 6: 35576, 2016.

88. Li RC, Ke S, Meng FK, Lu J, Zou XJ, He ZG, Wang WF and Fang MH: CiRS-7 promotes growth and metastasis of esophageal squamous cell carcinoma via regulation of miR-7/HOXB13 Cell Death Dis 9: 838, 2018

89. Xiao-Long M, Kun-Peng Z and Chun-Lin Z: Circular RNA circ_HIPK3 is down-regulated and suppresses cell proliferation, migration and invasion in osteosarcoma. J Cancer 9: 1856-1862, 2018.
90. Huang L, Chen M, Pan J and Yu W: Circular RNA circNASP modulates the malignant behaviors in osteosarcoma via miR-1253/FOXF1 pathway. Biochem Biophys Res Commun 500: 511-517, 2018

91. Song Y and Li J: Circular RNA hsa_circ_0001564 regulates osteosarcoma proliferation and apoptosis by acting miRNA sponge. Biochem Biophys Res Commun 495: 2369-2375, 2018.

92.Xia L, Wu L, Bao J, Li Q, Chen X, Xia H and Xia R: Circular RNA circ-CBFB promotes proliferation and inhibits apoptosis in chronic lymphocytic leukemia through regulating miR-607/FZD3/Wnt/ $\beta$-catenin pathway. Biochem Biophys Res Commun 503: 385-390, 2018.

93. Li S, Ma Y, Tan Y, Ma X, Zhao M, Chen B, Zhang R, Chen Z and Wang K: Profiling and functional analysis of circular RNAs in acute promyelocytic leukemia and their dynamic regulation during all-trans retinoic acid treatment. Cell Death Dis 9: 651, 2018.

94. Wu DM, Wen X, Han XR, Wang S, Wang YJ, Shen M, Fan SH, Zhang ZF, Shan Q, Li MQ, et al: Role of Circular RNA DLEU2 in Human Acute Myeloid Leukemia. Mol Cell Biol 38: pii: e00259-18, 2018.

95. Ma HB, Yao YN, Yu JJ, Chen XX and Li HF: Extensive profiling of circular RNAs and the potential regulatory role of circRNA-000284 in cell proliferation and invasion of cervical cancer via sponging miR-506. Am J Transl Res 10: 592-604, 2018.

96. Liu J, Wang D, Long Z, Liu J and Li W: CircRNA8924 promotes cervical cancer cell proliferation, migration and invasion by competitively binding to MiR-518d-5p /519-5p family and modulating the expression of CBX8. Cell Physiol Biochem 173-184, 2018.

97. Zhong Z, Huang M, Lv M, He Y, Duan C, Zhang L and Chen J: Circular RNA MYLK as a competing endogenous RNA promotes bladder cancer progression through modulating VEGFA/VEGFR2 signaling pathway. Cancer Lett 403: 305-317, 2017

98. Yang X, Yuan W, Tao J, Li P, Yang C, Deng X, Zhang X, Tang J, Han J, Wang J, et al: Identification of circular RNA signature in bladder cancer. J Cancer 8: 3456-3463, 2017.

99. Yang C, Yuan W, Yang X, Li P, Wang J, Han J, Tao J, Li P, Yang H, Lv Q and Zhang W: Circular RNA circ-ITCH inhibits bladder cancer progression by sponging miR-17/miR-224 and regulating p21, PTEN expression. Mol Cancer 17: 19, 2018.

100. Huang G, Li S, Yang N, Zou Y, Zheng D and Xiao T: Recent progress in circular RNAs in human cancers. Cancer Lett 404: 8-18, 2017.

101. Li Y, Zheng Q, Bao C, Li S, Guo W, Zhao J, Chen D, Gu J, He X and Huang S: Circular RNA is enriched and stable in exosomes: A promising biomarker for cancer diagnosis. Cell Res 25: 981-984, 2015.

102. Alhasan AA, Izuogu OG, Al-Balool HH, Steyn JS, Evans A, Colzani M, Ghevaert C, Mountford JC, Marenah L, Elliott DJ, et al: Circular RNA enrichment in platelets is a signature of transcriptome degradation. Blood 127: e1-e11, 2016.

103. Nicolet BP, Engels S, Aglialoro F, van den Akker E, von Lindern $\mathrm{M}$ and Wolkers MC: Circular RNA expression in human hematopoietic cells is widespread and cell-type specific. Nucleic Acids Res 46: 8168-8180, 2018

104. Geng Y, Jiang J and Wu C: Function and clinical significance of circRNAs in solid tumors. J Hematol Oncol 11: 98, 2018.

105. Wang T, Shigdar S, Shamaileh HA, Gantier MP, Yin W, Xiang D, Wang L, Zhou SF, Hou Y, Wang P, et al: Challenges and opportunities for siRNA-based cancer treatment. Cancer Lett 387: 77-83, 2017

106. Frazier KS: Antisense oligonucleotide therapies: The promise and the challenges from a toxicologic pathologist's perspective. Toxicol Pathol 43: 78-89, 2015.

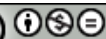

This work is licensed under a Creative Commons Attribution-NonCommercial-NoDerivatives 4.0 International (CC BY-NC-ND 4.0) License. 